Secondary acute leukaemia (s-ALL) is a destructive complication in patients who have been previously treated for other cancer. Secondary acute lymphoblastic leukaemia is rarely reported whereas secondary acute myeloid leukaemia is much more common. Chromosomal 11q23 abnormality, frequently detected in therapy-related acute myeloid leukaemia, is the most common cytogenetic alteration in secondary ALL too. However, s-ALL cases without 11q23 abnormality have rarely been described. Furthermore, there are only a few published medical reports describing occurrence of acute lymphoblastic leukaemia in multiple myeloma (MM) patients. We would like to present our experience with a patient with $M M$, who developed ALL without 11q23 abnormality, nine years after alkylating-agent containing treatment. The course of the disease was complicated by thrombosis that obstructed the possibility of effective treatment. In conclusion, it should be kept in mind that the development of a more aggressive neoplasm related to chemotherapy treatment as well as the inherent genetic instability of normal and abnormal lymphoid progenitors may affect overall survival of an indolent lymphoma patient.

Key words: multiple myeloma, secondary acute leukaemia, chemotherapy.

\section{Secondary acute lymphoblastic leukaemia in a multiple myeloma patient}

\author{
Jaroslaw Piszcz, Lukasz Bolkun, Edyta Cichocka, Janusz Kloczko
}

Department of Haematology, Medical University of Bialystok, Bialystok, Poland

\section{Introduction}

Secondary acute leukaemia (s-ALL) is a destructive complication in patients who have been previously treated for other cancer. Secondary acute lymphoblastic leukaemia is rarely reported whereas secondary acute myeloid leukaemia is much more common. Chromosomal 11q23 abnormality, frequently detected in therapy-related acute myeloid leukaemia, is the most common cytogenetic alteration in secondary acute lymphoblastic leukaemia too. However, s-ALL cases without 11q23 abnormality have rarely been described. Furthermore, there are only a few published medical reports describing occurrence of acute lymphoblastic leukaemia in multiple myeloma (MM) patients. We would like to present our experience with a patient with $M M$, who developed ALL without 11q23 abnormality, nine years after alkylating-agent containing treatment.

A 56-year-old woman who presented with fatigue and bone pain of the lumbar-sacral area was admitted to our hospital in July 2000. Serum immunoelectrophoresis showed increased monoclonal lgG $(1.9 \mathrm{G} / \mathrm{dl})$, and normal creatinine level. The bone marrow (BM) aspiration revealed $11.2 \%$ plasma cells and $51 \%$ infiltration by malignant plasma in trephine biopsy. Skeletal survey showed many lytic lesions of the skull and lumbar-sacral area. A diagnosis of MM was made. The patient was treated with seven cycles of the VCMP (vincristine, cyclophosphamide, melphalan, and prednisone) regimen. The evaluation of the response after treatment was partial remission. Due to the progression of MM the patient was treated with nine cycles of melphalan, prednisolone (dexamethasone) and thalidomide (MPT), from January 2004 to April 2005. The patient achieved partial remission. From May 2005 the patient was treated with thalidomide in monotherapy.

On September 2009 the patient was admitted to the Haematology Department due to complaints of oedema of the right lower limb and dyspnoea. The physical examination revealed enlargement of the spleen $10 \mathrm{~cm}$ below the costal margin, tachypnoea and pleural friction rub. The complete blood count showed white blood cell count (WBC) $30.77 \mathrm{G} / \mathrm{l}$, haemoglobin (HGB) $8.6 \mathrm{~g} / \mathrm{dl}$, platelet count (PLT) $80000 / \mathrm{ml}$ and $5 \%$ blastic cells in the peripheral smear. The BM was infiltrated by $37.8 \%$ blasts, immunophenotyped as B-cell lymphoblasts by flow cytometry (CD19+, CD20+, CD22+, HLA-DR+ CD10+) and negative antigen of myeloid and T-cell lineage. Results were consistent with acute lymphocytic leukaemia B common. Cytogenetics revealed normal karyotype (46,XX [20/20]) with negative fluorescence in situ hybridization (FISH) examination for mixed lineage leukaemia gene MLL rearrangement and BCR/ABL fusion gene. The serum immunoelectrophoresis showed the $\operatorname{lgG}$ monoclonal protein with level $0.36 \mathrm{~g} / \mathrm{dl}$. Doppler examination of the right lower limb confirmed deep venous thrombosis and CT pulmonary angiography revealed multiple filling defects of principal branches of the pulmonary arteries, due to pulmonary embolism. The vascular complications were treated with low molecular 
heparin in therapeutic doses. In view of all these conditions and the poor overall prognosis the patient was managed conservatively with steroids alone. After ten days of treatment the patient died due to acute cardio-pulmonary failure.

Therapy-related acute leukaemia is a heterogeneous disease that may occur especially after treatment containing an alkylating agent/radiotherapy and/or topoisomerase ॥ inhibitor [1]. The alkylating agent-related subgroup is characterized by having a mean latency period of 5 to 7 years and antecedent myelodysplasia with chromosomal aberration typical for this state [2]. These alkylating agent-related acute leukaemias are mostly classified as acute myeloid leukaemia (AML) [3]. On the other hand, DNA topoisomerase II inhibitors cause secondary leukaemias with relatively short latent periods (1-5 years) without antecedent myelodysplasia. The most common characteristic chromosomal aberrations are translocations involving 11q23, the MLL gene locus. Most of the leukaemias are diagnosed as AML [4]. Therapy-related acute lymphoblastic leukaemia, which represents approximately $12 \%$ of all therapy-related acute leukaemias and $1.2 \%$ to $4 \%$ of adult $\operatorname{ALLs}[5,6]$, is seen much less frequently than therapy-related AML. The most common chromosomal abnormalities in s-ALL affect the $M L L$ gene (11q23); others are extremely rare but have been reported previously. In patients with multiple myeloma, the risk of developing secondary AML has been calculated to be $3-5 \%$ at 3 and $10-15 \%$ at 10 years after treatment with alkylating agent therapy. On the other hand, secondary acute lymphoblastic leukaemia is rare, occurring in about $0.5-1 \%$ of treated patients [7].

Ueda et al. (2009) reported the case of a multiple myeloma patient, treated with an alkylating agent, that terminated in acute lymphoblastic leukaemia with gene MLL aberration one year after autologous transplantation. The authors performed IgH gene rearrangement studies on genomic DNA extracted from the BM aspirates at the time of diagnosis of MM and that of ALL, which showed different monoclonal bands on DNA extracted from both samples [8]. Based on this, they concluded that previous MM and S-ALL were derived from different lymphoid clones. The authors postulated that MLL modification may lead to ALL in the multi-step tumorigenesis process [9]. However, that kind instability in a lymphoid progenitor of MM cells in vivo or in vitro has not yet been confirmed. Furthermore, the development of s-ALL without MLL gene aberration, 3 years after tandem autologous stem cell transplantation due to MM, was previously described by Lau et al. (2005). The presented patient was treated with the VAD regimen (vincristine, doxorubicin, dexamethasone) as induction therapy, and with alkylating agents (cyclophosphamide and melphalan as a mobilization and a conditioning treatment respectively). The authors confirmed that two separate monoclonal B-cell populations were involved in the pathogenesis of these two lymphoid malignancies at two different time points [10]. Recently, Chen et al. (2010) described 6 adults with secondary treatment related ALL without 11q23 abnormalities following various treatment regimens for primary malignancies (2 MM patients included). They also reviewed $48 \mathrm{~s}$-ALL cases, with complete chromosomal karyotyping, reported in the literature from 1992 to 2007 (13 patients with haematological malignancy, num- ber of MM not specified). In the 48 cases, an 11q23 abnormality involving the MLL gene locus was the predominant chromosomal aberration (67\%), and 8\% had a normal karyotype. The two described cases of MM patients previously treated with anthracycline agents revealed s-ALL 78 and 60 months after diagnosis of primary malignancy and had no 11q23 abnormalities. The authors showed that s-ALL cases with an 11q23 abnormality compared to cases without an 11q23 abnormality had a longer latency period (median, 36 vs. 19 months) and a different primary malignancy spectrum [11].

Our patient had a normal karyotype and received only an alkylating agent in MM treatment. The latency period was 102 months from diagnosis of MM and was comparable with the mean latency time described in the literature for s-ALL related to alkylating agents. The vascular incident that complicated the course of disease obstructed the possibility of aggressive and more effective treatment. In our clinical practice we have to remember that s-ALL may complicate the course of other indolent haematological malignancies. The development of a more aggressive neoplasm could be related to applied chemotherapy as well as the inherent genetic instability of normal and abnormal lymphoid progenitors.

The authors declare no conflict of interest.

\section{References}

1. Vardiman JW, Thiele J, Arber DA, et al. Acute myeloid leukaemia $(\mathrm{AML})$ and related precursor neoplasms. In: Swerdlow SH, Campo E, Harris NL, et al. (eds.). WHO Classification of Tumours of Haematopoietic and Lymphoid Tissues. Lyon, France: IARC Press; 2008: 110-147. WHO Classification of Tumours; vol. 2.

2. Hawkins MM, Wilson LM, Stovall MA, Marsden HB, Potok MH, Kingston JE, Chessells JM. Epipodophyllotoxins, alkylating agents, and radiation and risk of secondary leukaemia after childhood cancer. BMJ 1992; 304: 951-8

3. Zhang Y, Poetsch M, Weber-Matthiesen K, et al. Secondary acute leukaemias with 11q23 rearrangement: clinical, cytogenetic, FISH and FICTION studies. Br J Haematol 1996; 92: 673-80.

4. Cortes J, O'Brien S, Kantarjian $\mathrm{H}$, et al. Abnormalities in the long arm of chromosome 11 (11q) in patients with de novo and secondary acute myelogenous leukemias and myelodysplastic syndromes. Leukemia 1994; 8: 2174-8.

5. Ishizawa S, Slovak ML, Popplewell L, Bedell V, Wrede JE, Carter NH, Snyder DS, Arber DA. High frequency of pro-B acute lymphoblastic leukemia in adults with secondary leukemia with 11q23 abnormalities. Leukemia 2003; 17: 1091-5.

6. Pagano L, Pulsoni A, Tosti ME, et al. Acute lymphoblastic leukaemia occurring as second malignancy: report of the GIMEMA archive of adult acute leukaemia. Gruppo Italiano Malattie Ematologiche Maligne dell'Adulto. Br J Haematol 1999; 106: 1037-40.

7. Leone G, Voso MT, Sica S, Morosetti R, Pagano L. Therapy-related leukemias: susceptibility, prevention and treatment. Leukemia Lymphoma 2001; 41: 255-76.

8. Ueda K, Yamamoto G, Shinohara A, Hangaishi A, Kurokawa M. Early onset of acute lymphoblastic leukemia with MLL rearrangement after autologous stem cell transplantation for multiple myeloma. Ann Hematol 2009; 88: 813-4.

9. Pedersen-Bjergaard J. Insights into leukemogenesis from therapyrelated leukaemia. N Engl J Med 2005; 352: 1591-4.

10. Lau LG, Tan LK, Koay ES, Liu TC. Acute lymphoblastic leukemia after tandem autologous stem cell transplantations for multiple myeloma. Leukemia 2005; 19: 299-301. 
11. Chen W, Wang E, Lu Y, Gaal KK, Huang O. Therapy-related acute lymphoblastic leukemia without 11q23 abnormality: report of six cases and a literature review. Am J Clin Pathol 2010; 133: 75-82.

\section{Address for correspondence}

Jaroslaw Piszcz MD, PhD

Department of Haematology

Medical University of Bialystok

24 A Sklodowskiej-Curie

15-276 Bialystok, Poland

phone +48601311843

fax +48857447004

e-mail: jaroslaw.piszcz@gmail.com

Submitted: $\quad 19.04 .2012$

Accepted: $\quad 20.05 .2012$ 